\title{
Statistical Study Regarding the Presence of Gram Bacteria in Patients with Cardiovascular Disease and Periodontal Disease
}

\author{
Ioana VOINESCU ${ }^{1,2}$, Dumitru FERECHIDE²
}

\begin{abstract}
Pathogenic bacteria, originating in the inflamed periodontium, can make their way into the body through the vascular system, either by entering the bloodstream or lymph directly or as internalized particles of immune cells. This research aims to investigate the existence of a potential connection between two pathologies - periodontitis and ischemic heart disease - by utilizing a DNA-DNA hybridization technique to highlight the presence of seven periodontal pathogens: A. actinomycetemcomitans, P. gingivalis, P. endodontalis, P. intermedia, T. forsythia, C. rectus and F. Nucleatum. The study was conducted over a period of 3 years on a sample consisting of 119 patients with cardiovascular disease and periodontal disease, divided into 3 groups by pathologies, as follows: the first group consisted of 31 patients with ischemic cardiomyopathy, the second group consisted of 43 patients with periodontal disease and ischemic cardiomyopathy, and the third group consisted of 45 patients with periodontal disease.

The results show the possibility of a correlation between the composition of the bacterial flora in the group of patients with cardiac disease and those with associated pathologies.

In conclusion, we may state that periodontitis may be seen as a risk factor in heart disease, which may certify the possibility of an etiological connection between the two pathologies.
\end{abstract}

Keywords: periodontal disease, ischemic cardiomyopathy, gram-negative bacteria.

' Faculty of Midwives and Nursing, „Carol Davila" University of Medicine and Pharmacy, Bucharest, Romania

2 Faculty of Medicine, „Carol Davila" University of Medicine and Pharmacy, Bucharest, Romania

\section{Corresponding author.}

Ioana VOINESCU, Faculty of Midwives and Nursing, "Carol Davila” University of Medicine and Pharmacy, Bucharest, Romania E-mail: ioana.voinescu@umfcd.ro 


\section{INTRODUCTION}

Oral infections occur frequently in humans and often lead to chronic inflammation that affects teeth, the gingival tissue around teeth and the support structures of teeth. These chronic inflammations are sustained by bacteria populating the surface of teeth, in the form of biofilm. They may infect the gum and may trigger an immune response in the gingival tissue ${ }^{2}$. Pathogenic bacteria, originating in the inflamed periodontium, can make their way into the body through the vascular system, either by entering the bloodstream or lymph directly or as internalized particles of immune cells ${ }^{1}$. The periodontal biofilm produces an inflammatory response, leading to the gradual destruction of surrounding tissues. Periodontitis, along with local damage, determines an inflammatory response in the host and triggers systemic inflammation ${ }^{3}$.

The periodontal biofilm includes various species of gram-negative and gram-positive bacteria. Periodontal pockets, which form as a result of periodontal disease, provide a perfect breeding ground for these bacteria to grow and multiply.

Periodontal bacteria involved in bacteremia include pathogens such as: Aggregatibacter actinomycetemcomitans, Porphyromonas gingivalis, Tannerella forsythia, Treponema denticola and Fusobacterium nucleatum, and are gram-negative bacteria ${ }^{5}$. These enter the bloodstream directly and may produce endotoxins that create inflammatory cytokines which alter the molecular adhesion of the endothelium, potentially leading to prothrombotic events. All these are risk factors that may aggravate the presence of cardiovascular disease ${ }^{6}$.

To examine the probability for oral bacteria to be associated with atherosclerosis, the present research was focused on the detection of molecular markers utilizing the polymerase chain reaction method (RT-PCR), well known for the detection of oral bacteria $\mathrm{DNA}^{7}$.

Data in the specialized literature show Chlamydia pneumoniae has been detected in atherosclerotic plaques ${ }^{8}$, Porphyromonas gingivalis in some aorta samples $^{9}$, Aggregatibacter actinomycetemcomitans in some aortic aneurysm samples ${ }^{10}$, and a considerably high level of Aggregatibacter actinomycetemcomitans has been detected in human atherosclerotic tissue ${ }^{11}$. Through cellular motility, Treponema denticola can infiltrate between gingival cells and the bloodstream. Porphyromonas gingivalis has been identified using the RT-PCR technique as a significant marker of periodontal disease ${ }^{12}$.

\section{MATERIAL AND METHOD}

The study was conducted on a sample of 119 patients with cardiac disease and periodontal disease. The patients were divided into 3 groups following the application of selection and exclusion criteria: the first group consisted of 31 patients with ischemic cardiomyopathy, the second group consisted of 43 patients with periodontal disease and ischemic cardiomyopathy, and the third group consisted of 45 patients with periodontal disease. The age of the patients examined ranged between 30 and 68 years. The study was conducted over a period of 3 years.

Selection criteria were: written consent from patients, patients diagnosed with ischemic cardiomyopathy, patients diagnosed with periodontal disease. Exclusion criteria were: lack of written consent from patients, incomplete data in patient diagnosis, patients with malignant tumor pathology, patients diagnosed with mental illness, on immunosuppressant medication, biphosphonate treatments, patients with immunemediated inflammatory disease, dysmetabolic syndrome, endocrine disorders, neurological, hepatic, hematological, renal disease, history or neoplastic diseases.

Bacterial samples were collected from the gingival sulcus todetect the bacteriallevels of thesevenperiodontal pathogens examined: $A$. actinomycetemcomitans, $P$. gingivalis, $P$. endodontalis, $P$. intermedia, T. forsythia, $C$. rectus and $F$. nucleatum, samples that were analyzed by utilizing the DNA-DNA hybridization technique.

The bacterial samples were collected from the dental units presenting the deepest periodontal pockets for each quadrant. Thus, we have selected the proximal surfaces for molars from all quadrants. The sample collection areas were first isolated using suctioning and cotton pellets. Using a sterile probe, the sample was collected with a paper cone from the sampling kit. The probe was maintained for 10 seconds in each collection point, then it was introduced into a transfer tube and immediately sent to the laboratory (per instructions, a maximum of 4 samples may be collected from different periodontal pockets).

For the statistical analysis of bacterial presence we carried out a non-parametric dichotomous valuation of the lab results where we established a score of 0 for undetectable bacterial species, a score of 1 for low level of bacterial presence and a score of 2 for high level of bacterial presence. Associations between continuous variables were determined using a linear regression 
model, the Pearson Chi correlation test where $\mathrm{p}<0.1$ was considered statistically relevant.

\section{RESULTS}

Investigations of the composition of the bacterial flora in periodontal pockets in the three groups studied showed significant differences in bacterial levels between the group with periodontitis and the group with cardiomyopathy for the species Aggregatibacter actinomycetemcomitans, Tannerellaforsythia, Campylobacter rectus, Prevotella nigrescens and Capnocytophaga sputigena. Except for the first species mentioned, these appear in high concentrations in group 3.

In the synthesis of bacterial concentrations by pathology we note that group 1 registers the highest concentrations of Fusobacterium nucleatum, Aggregatibacter actinomycetemcomitans, Porphyromonas gingivalis, Eikenella corrodens, Capnocytophaga sputigena, Capnocytophaga sputigena and the lowest concentrations of Campylobacter rectus; group 2 shows high levels of Eikenella corrodens, Fusobacterium nucleatum, Capnocytophaga ochracea and gingivalis and the lowest levels of Aggregatibacter actinomycetemcomitans; group 3 shows significant levels for all bacteria analyzed, except for Aggregatibacter actinomycetemcomitans.

In groups 1 and 2, the Pearson index $\mathrm{r}=0.372$ shows the possibility of this correlation in $13.87 \%$ of the cases (Graph 1.1). In the group with both pathologies, the Pearson index $r=0.60774$ shows a higher degree of correlation for $36.9 \%$ of the cases (Graph 1.2). Groups 1 and 3 show the lowest degree of correlation with a negative value for the Pearson index $\mathrm{r}=-0.12234, \mathrm{y}=-0.0921 \mathrm{x}+0.9131 \mathrm{R}^{2}=0.015$, which signifies a substantial difference in the composition of the bacterial flora in the group with cardiomyopathy and the group with periodontitis (Graph 1.3).

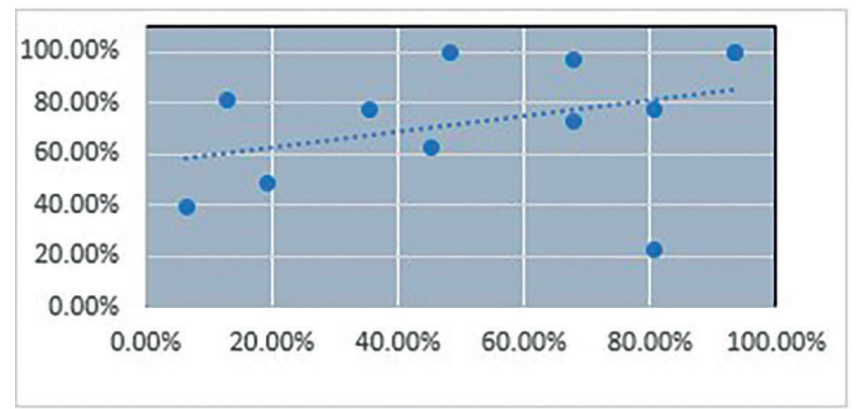

Graph 1.1. Correlation of groups 1 and 2

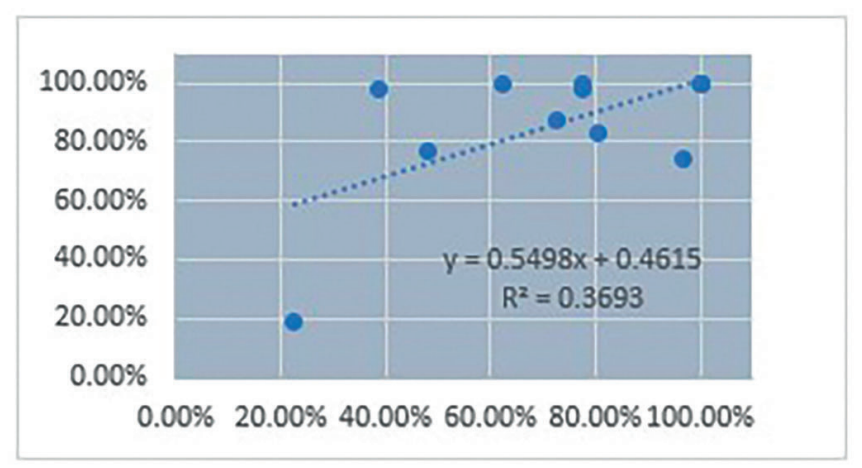

Graph 1.2. Correlation of groups 2 and 3

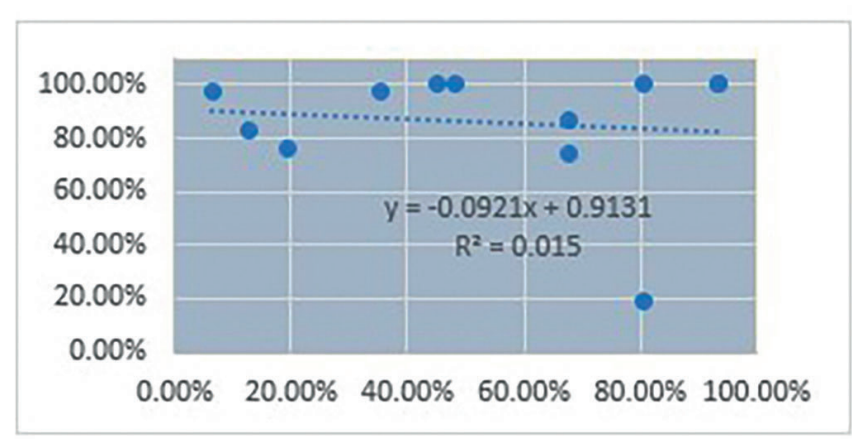

Graph 1.3. Correlation of groups 1 and 3

\section{DISCUSSION}

Porfiromonas gingivalis was detected in a high percentage of $86.2 \%$ in group 3 compared with the percentage found in Boutaga's 2006 study ${ }^{12}$ utilizing the same technique on periodontitis patients (45.5\%). This result is consistent with pathogenic characteristics of this species that suggest the ability to induce a systemic inflammatory response ${ }^{13}$. Porfiromonas gingivalis was also highlighted in Griffien's study ${ }^{14}$, which used the same PCR technique and found an $\mathrm{OR}=11.2$ for adult patients with periodontitis.

The fact that a pathogen can be frequently detected in healthy subjects suggests that not all humans are equally susceptible to developing cardiovascular disease ${ }^{15}$. The presence of a pathogen species is important for the development of periodontitis. But for the periodontal disease to be manifested, that pathogen must exceed a threshold for the host ${ }^{16}$. The low prevalence of $A$. actinomycetemcomitans and Porfiromonas Gingivalis in patients with no periodontal disease supports the hypothesis that both species can be exogenous oral pathogens.

Tannerella forsythia is found in $12.9 \%$ of patients with cardiomyopathy, a low percentage compared with 
other studies on healthy patients. Our results show a significant statistical difference between groups 1 and 2 , and 1 and 3, respectively, a result that is consistent with Boutaga's study ${ }^{13}$, which concludes that Porfiromonas gingivalis and T. forsythia are prominent markers for periodontitis in adult patients.

Treponema denticola is strongly associated with the development of periodontal disease, with pathogenic effects in low concentrations, associated with aggressive forms of disease. It is present in all three test groups, found in higher percentages in the periodontitis group and the group with associated pathologies, which is consistent with other results in the literature ${ }^{12}$.

Prevotella intermedia was detected with a high prevalence in group $3(76.5 \%)$, similar to other results mentioned in the literature ${ }^{17}$. The other two groups had lower percentages. The statistical analysis showed significant difference between single-pathology groups, which, considering the conclusion of Sparh's study ${ }^{18}$, may indicate this bacteria as a potential risk for ischemic cardiomyopathy. Prevotella nigrescens shows a significant difference between groups 2 and 3, making it a potential marker of affected cardiac tissue.

Fusobacterium did not show statistical differences between groups although it was present in high concentrations in all 3 groups. Because the Fusobacterium genus includes several (sub)species ${ }^{19}$, pathogenicity may vary depending on the (sub)species.

Among bacteria with moderate pathogenicity, Campylobacter rectus had a high prevalence in group 3 (97.8\%), higher compared with the group of patients with associated pathologies and compared with the group of cardiac patients, with statistical differences between groups 1 and 3, and 2 and 3. The association of this bacteria with periodontal disease is evident, the result is correlated with Llijestrand's study ${ }^{20}$. Ashimoto and co. suggested that C.ampylobacter rectus is a species that is part of the normal oral flora and may be considered an endogenous pathogen that occasionally contributes to the development of periodontal disease ${ }^{21}$.

Capnocytophaga ochracea, sputigena and gingivalis are known as having low association with periodontal disease and are often identified in active sites. In the group of patients with periodontitis their prevalence is maximal but it showed high prevalence in the group with associated pathologies as well. There are, however, statistically significant differences for Capnocytophaga ochracea between groups 1 and 2, and 1 and 3, which certifies that its occurrence is related to periodontium damage, while for Capnocytophaga gingivalis the difference occurs between the group with associated pathologies and the group with periodontal disease, which may be a clue for cardiac damage.

Capnocytophaga gingivalis, Porphyromonas gingivalis, Eikenella corrodens, Fusobacterium nucleatum show the smallest differences, which suggests they play an insignificant role in changes occurring at the periodontal level.

A statistically significant correlation could not be established between the composition of the oral bacterial flora in groups of patients with cardiovascular disease and periodontal disease, which shows that the two types of pathologies induce specific modifications. Correlation between the group of patients with cardiovascular disease and the group of patients with both pathologies is very weak, while in $36.9 \%$ of cases a correlation can be established between the flora in the group with both pathologies and the flora found in the group of periodontal patients, which may certify the possibility of an etiological connection between the two pathologies.

\section{CONCLUSIONS}

Factors that differentiate the group of patients with periodontal disease from the group of patients with both pathologies are: age, a history of smoking, the average number of teeth present, degree of periodontal damage, concentration of Campylobacter rectus, Prevotella nigrescens, Capnocitofaga sputigena, Tannerella forsythia, Capnocytophaga ochracea.

In $36.9 \%$ of cases a correlation can be established between the flora in the group with both pathologies and the flora found in the group of periodontal patients. Our conclusions suggest that periodontitis may be seen as a risk factor in heart disease, which may certify the possibility of an etiological connection between the two pathologies.

Compliance with ethics requirements: The authors declare no conflict of interest regarding this article. The authors declare that all the procedures and experiments of this study respect the ethical standards in the Helsinki Declaration of 1975, as revised in 2008(5) and the national law. Informed consent was obtained from the patient described in the clinical case and his parents. 


\section{References}

1. Ali Cekici, Alpdogan Kantarci, Hatice Hasturk, and Thomas E. Van Dyke. Inflammatory and immune pathways in the pathogenesis of periodontal disease. Periodontol 2000. 2014; 64(1): 57-80. doi:10.1111/prd.12002.

1. Loe, H.; Theilade, E.; Jensen, S.B. Experimental gingivitis in man. J. Periodontol. 1965, 36, 177-187. doi.org/10.1902/ jop.1965.36.3.177.

2. D'Aiuto F, Parkar M, Andreou G, Suvan J, Brett PM, Ready D et al. Periodontitis and systemic inflammation: control of the local infection is associated with a reduction in serum inflammatory markers. J Dent Res 2004; 83:156-60. doi: 10.1177/154405910408300214.

3. Alaluusua S., Asikainen S.. Detection and distribution of Actinobacillus actinomycetemcomitans in the primary dentition. 1998, J Periodontol 59:504-507. doi: 10.1177/154405910408300214.

4. Viviane Aparecida Arenas Rodrigues, Erica Dorigatti de Avila, Viviane Nakano, Mario Julio Avila-Campos. Qualitative, quantitative and genotypic evaluation of Aggregatibacter actinomycetemcomitans and Fusobacterium nucleatum isolated from individuals with different periodontal clinical conditions. Anaerobe 2018. 52:50-58. doi: 10.1016/j.anaerobe.2018.05.015.

5. J., V. I. Haraszthy, M. Trevisan, M. Zeid, R. J. Genco. Identification of peiodontal pathogens in atheromatous plaques. 2000; 71(10):1554-60. doi: 10.1902/jop.2000.71.10.1554.

6. Watanabe K. Frommel TO: Porphyromonas gingivaiis, Actinobaciilus actinomycetemeomitans and Treponema denticoia detection in oral plaque samples using the polymerase chain reaction. J Clin Periodontoi 1996: 23: 212-219. doi: 10.1111/j.1600-051x.1996. tb02078.x.

7. M Maass, CBartels, PM Engel, U Mamat, HH Sievers. Endovascular presence of viable Chlamydia pneumoniae is a common phenomenon in coronary artery disease. J Am Coll Cardiol 1998, 15;31(4):827-32. doi: 10.1016/s0735-1097(98)00016-3.

8. Stelzel M, Conrads G, Pankuweit $S$ et al. Detection of Porphyromonas gingivalis DNA in aortic tissue by PCR. J Periodontol 2002; 73: 868- 870. doi: 10.1902/jop.2002.73.8.868.

9. Marques da Silva RDA, Caugant PS, Lingaas O, Geiran L, Tronstad Olsen I. Detection of Actinobacillus actinomycetemcomitans but not bacteria of the red complex in aortic aneurysms by multiplex polymerase chain reaction. J Periodontol 2005; 76: 590- 594. doi: 10.1902/jop.2005.76.4.590.

10. Kozarov EV, Dorn BR, Shelburne CE, Dunn WA Jr, Progulske-Fox A. Human atherosclerotic plaque contains viable invasive Actinobacillus actinomycetemcomitans and Porphyromonas gingivalis. Arterioscler Thromb Vasc Biol 2005; 25: 17- 18. doi: 10.1161/01.ATV.0000155018.67835.1a.
11. K. Boutaga, A. J. van Winkelhoff, C. M. J. E. VandenbrouckeGrauls, H. M. Savelkoul. Comparison of real-time PCR and culture for detection of Porphynomonas gingivalis in subgingival plaque samples. Jornal of Clinical Microbiology. 2003; 41(11):49504954. doi: 10.1128/JCM.41.11.4950-4954.2003.

12. M. C. Herzberg. Coagulation and thrombosis in cardiovascular disease: plausible contributions of infectious agents. Annals of Periodontology; 6(1):16-19. doi: 10.1902/annals.2001.6.1.16.

13. A. L. Griffin, M. R. Becker, S. R. Lyions, M. L. Moeschberger, E. J. Leys. Prevalence of Porphyromonas gingivalis and periodontal health status. Journal of clinical microbiology. 1998; 36(11):32393242. doi: 10.1128/JCM.36.11.3239-3242.1998.

14. DW. Holborow. The Community Periodontal Index of Treatment Needs-uses and abuses? N Z Dent J. 1998; 94(417):120-121. PMID: 9775647.

15. S. S. Socransky, A. D. Haffajee. The bacterial etiology of destructive periodontal disease: current concepts. Journal of periodontology. 1992; 63(45):322-331.doi: 10.1902/jop.1992.63.4s.322.

16. M. Okada, F. Hayashi, N. Nagasaka. PCR detection of 5 putative periodontal pathogens in dental plaque samples from children 2 to 12 years of age. Journal of clinical periodontology. 2001; 28(6):576-582. doi: 10.1034/j.1600-051x.2001.028006576.x

17. A. Spahr, E. Klein, N. Khuseyinova, C. boeckh, R. Muche, M. Kunze, D. Rothenbacher, G. Pezeshki, A. Hoffmeister, W. Koenig. Role of periodontal bacteria and importance of total pathogen burden in the coronary event and periodontal disease (CORODONT) study. Arch Intern Med. 2006; 166(5):554-559. doi: 10.1001/ archinte.166.5.554.

18. Diane M. Citron. Update on the Taxonomy and Clinical Aspects of the Genus Fusobacterium Clinical Infectious Diseases 2002. Volume 35(1): S22-S27. doi.org/10.1086/341916.

19. J. M. Liljestrand, S. Paju, M. Pietiäinen, K. Buhlin, G. R. Perrson, M. S. Nieminen, J. Sinisalo, P. Mäntylä, P. Pussinen. Immunologic burden links periodontitis to acute coronary syndrome. Atherosclerosis. 2018; 268:177-184. doi: 10.1016/j.atherosclerosis.2017.12.007.

20. A. Ashimoto, C. Chen, I. Bakker, J. Slots. Polymerase chain reaction detection of 8 putative periodontal pathogens in subgingival plaque of gingivitis and advanced periodontitis lesions. Oral Microbiology and Immunology. 1996; 11(4):266-273. doi: 10.1111/j.1399-302x.1996.tb00180.x. 